\title{
Do most lymphocytes in humans really reside in the gut?
}

\author{
Vitaly V. Ganusov ${ }^{1,2}$ and Rob J. De Boer ${ }^{1}$ \\ ${ }^{1}$ Theoretical Biology, Utrecht University, Padualaan 8, $3584 \mathrm{CH}$, Utrecht, The Netherlands \\ ${ }^{2}$ On a leave of absence from Krasnoyarsk Science Center and Institute of Biophysics, Akademgorodok, Krasnoyarsk, Russia 660036
}

\begin{abstract}
It is widely believed that the gut, and particularly the lamina propria (LP) of the gut, contains most of the lymphocytes in humans. The strong depletion of CD4 ${ }^{+}$ $T$ cells from the gut LP of HIV-infected patients was, therefore, suggested to be such a large, irreversible insult that it could explain HIV disease progression. However, reviewing data from different mammalian species, we found that only $\mathbf{5 \%}-\mathbf{2 0} \%$ of all lymphocytes reside in the gut, and that only $1 \%-9 \%$ of the total lymphocyte number is located in the gut LP. Our findings suggest that spleen and lymph nodes, rather than the gut, are the largest immune compartments in mammals.
\end{abstract}

\section{Introduction}

Despite 20 years of research, we still fail to understand how human immunodeficiency virus (HIV) causes acquired immunodeficiency syndrome (AIDS). Recent studies have highlighted the importance of early depletion of $\mathrm{CD} 4^{+}$ $\mathrm{T}$ cells in the gut occurring after infection of humans with HIV-1 [1-6] and rhesus macaques with SIV [7-11]. These authors have observed a rapid and large reduction in the fraction of $\mathrm{CD} 4^{+} \mathrm{T}$ cells in the lamina propria (LP) of the gut, and several follow-up papers argued that this "first cut" is important for disease progression [12-16]. It is not clear, however, why such a massive and rapid depletion of LP lymphocytes, which is believed to be only slowly (if ever) reversible $[17,18]$, would not generally result in a fast progression to AIDS.

The presumed importance of the depletion of $\mathrm{CD} 4^{+}$ $\mathrm{T}$ cells is at least partially owing to the widespread belief that the majority of $\mathrm{T}$ cells in mammals, including monkeys and humans, reside in the gut-associated lymphoid tissue (GALT) and in the LP of the gut $[8,10,19-21]$. During the chronic phase of HIV or SIV infection, a homeostatic response attempts to compensate for the ongoing depletion of $\mathrm{CD}^{+} \mathrm{T}$ cells in the gut [22]. If the gut indeed contains the majority of $\mathrm{CD}^{+} \mathrm{T}$ cells, it is easy to imagine that the magnitude of the homeostatic response would be too large to be sustained over long periods of time [23].

It seems that the belief that the gut contains most of the body lymphocytes stems from at least two observations. First, the absorbing surface area of the human gut is thought to be extremely large (e.g. Haase [24] cited an area of $400 \mathrm{~m}^{2}$ ), and many lymphocytes are expected to be present in the gut LP (i.e. underneath the epithelium).

Corresponding author: Ganusov, V.V. (v.v.ganusov@uu.nl).

Available online 26 October 2007.
However, the above-stated estimate of the gut surface includes microvilli, which do not harbor any lymphocytes; the surface area of the gut excluding microvilli is much smaller, on the order of $2-10 \mathrm{~m}^{2}[25,26]$. Second, it has been estimated that up to $80 \%$ of all IgA-producing plasma $\mathrm{B}$ cells in mice and humans are located in the gut, arguing for a large population of lymphocytes at this site [27,28]. However, antibody-secreting cells constitute only a small fraction of all lymphocytes in the body $[27,29]$.

Surprisingly, most of the widely cited literature claiming that the gut is the largest immune compartment of humans neither has information on the total number of lymphocytes nor on the distribution of B- and T-cell numbers in humans [19-21]. Reviewing studies estimating the total number of lymphocytes in the body and in the gut in several mammal species, we found that only a small fraction of lymphocytes resides in the gut LP. On average, only $\sim 5 \%$ of the entire lymphocyte population resides in the gut LP, and $\sim 12 \%$ of all lymphocytes reside in the gut. This finding challenges the current belief that the majority of $\mathrm{T}$ cells are located in the gut, and thus questions the importance of the depletion of LP $\mathrm{CD}^{+} \mathrm{T}$ lymphocytes in determining the progression to AIDS.

\section{Do most lymphocytes in mammals reside in the gut?}

According to the standard nomenclature of the Society for Mucosal Immunology, the immune system of the gut can be divided into inductive sites (such as GALT) and effector sites (such as gut LP) [30]. The GALT includes Peyer's patches (PPs), isolated lymphoid follicles (ILFs), and the appendix. Effector sites mainly include LP (with lamina propria lymphocytes [LPLs]) and surface epithelia (with intraepithelial lymphocytes [IELs]) [19,30]. For simplicity, we refer to lymphocytes found in the inductive and effector sites of the gut and in draining gut mesenteric lymph nodes (MLNs) as "gut lymphocytes." To our knowledge, there are no documented studies that directly estimate the total numbers of T and B lymphocytes in humans. Moreover, there is only limited information on the distribution, phenotype, and total numbers of lymphocytes in several species of mammals (see below). In an extensive review, Trepel [29] listed the number of lymphocytes found in several lymphoid organs of rats and humans and provided one of the first estimates of the total number of lymphocytes found in lymphoid tissues of rats. By extrapolating the distribution and the total number of lymphocytes in rats to humans, Trepel [29] estimated that a young adult human has, on average, $4.6 \times 10^{11}$ 


\section{Box 1. Distribution and total number of lymphocytes in man}

Estimates of the total number of lymphocytes in several lymphoid organs of humans have been summarized in the review of Trepel ([29], see Table 1). The total number of lymph nodes and their sizes are known in rats, but not in humans [29]. To calculate the number of lymphocytes residing in lymph nodes of humans, an extrapolation was made [29]. First, Trepel [29] summarized documented estimates of lymphocyte numbers and their density per kilogram of body weight for several lymphoid organs of rats and humans (such as blood, spleen, Peyer's patches, thymus, and bone marrow). This resulted in an average of $15.2 \times 10^{9}$ and $2.9 \times 10^{9}$ of lymphocytes per kilogram of body weight for rats and humans, respectively $(5: 1$ ratio). The total number of lymphocytes residing in primary and secondary lymphoid organs, including all lymph nodes of a $200 \mathrm{~g}$ rat, is $4.9 \times 10^{9}$ cells [29]. The average lymphocyte density in a $200 \mathrm{~g}$ rat is then $24.5 \times 10^{9}$ lymphocytes per kilogram of body weight. Using the ratio of 5:1 for lymphocyte density in rats and humans given above, and assuming that the ratio of lymphoid tissue to body weight in rats and humans is similar, one estimate of the number of lymphocytes in all lymphoid tissues of a $70 \mathrm{~kg}$ human is $24.5 \times 10^{9} / 5 \times 70=332 \times 10^{9}$ cells. In rats, $2.2 \%$ of all lymphocytes are found in the blood. The number of lymphocytes in the blood of humans is $10 \times 10^{9}$ cells (Table 1 ), and assuming that, also, $2.2 \%$ of all lymphocytes in humans reside in the blood, the second estimate of the total number of lymphocytes in all lymphoid tissues in humans is $10 \times 10^{9} / 0.022=450 \times 10^{9}$ cells. The average of the two estimates stated above is then $\sim 390 \times 10^{9}$ lymphocytes. This estimate, however, does not include lymphocytes found in nonlymphoid tissues such as the LP of the gut or the lung. Kindred [43] found that the LP of the gut in rats contains $3 \times$ more lymphocytes than the blood, which, for humans, would translate into $30 \times 10^{9}$ lymphocytes in the gut LP. The same number of lymphocytes is assumed to be present in the LP of the lung ( $30 \times 10^{9}$ cells), and $10 \times 10^{9}$ lymphocytes were estimated to be in other nonlymphoid organs [29]. The total number of lymphocytes in a young adult human $(70 \mathrm{~kg})$ was thus estimated to be $4.6 \times 10^{11}$ cells [29].

lymphocytes (Box 1 and Table 1). Westermann and Pabst [31] extended this study with the percentages of lymphocyte subsets (B or T, and CD4 or CD8) in different lymphoid tissues of humans. Taken together, these two studies suggest that LP lymphocytes constitute only a small fraction (6.5\%), whereas gut lymphocytes (including LP, PP, and ILF lymphocytes) constitute $\sim 11 \%$ of all lymphocytes in a human.

Our analysis of the distribution and the total number of lymphocytes in several mammalian species, including

Table 1. Estimates of the total number of lymphocytes in different organs of humans

\begin{tabular}{|lll|}
\hline Tissue & $\begin{array}{l}\text { Total cell } \\
\text { number }\left(\times \mathbf{1 0}^{\mathbf{9}}\right)\end{array}$ & $\begin{array}{l}\text { Percent } \\
\text { of total }\end{array}$ \\
\hline Blood & 10 & 2.2 \\
Lymph nodes & 190 & 41.2 \\
Spleen & 70 & 15.2 \\
Peyer's patches & 20 & 4.3 \\
Thymus & 50 & 10.8 \\
Bone marrow & 50 & 10.8 \\
Lamina propria of the gut & 30 & 6.5 \\
Lamina propria of the lung & 30 & 6.5 \\
Other tissues & 10 & 2.2 \\
Total number & $\mathbf{4 6 0}$ & $\mathbf{1 0 0}$ \\
\hline
\end{tabular}

An estimate of the total number of lymphocytes in lymph nodes includes cells found both in peripheral and mucosal lymph nodes [30]. An estimate of the lymphocyte number in Peyer's patches also includes lymphocytes found in isolated lymphoid follicles. Importantly, the estimate of the number of lymphocytes found in the thymus include immature lymphocytes and will depend dramatically on the age of a person owing to thymic involution; an estimate of $4.6 \times 10^{11}$ mature lymphocytes in a human should therefore be treated as approximate. The table is adapted from [29].

\section{Box 2. Estimating the total number of gut lymphocytes}

An estimate of the total number of lymphocytes in the gut LP, or its percentage of the total body lymphocyte number, was calculated in two ways. In some studies, the percentage was calculated by using estimates provided for the total number of lymphocytes in the body and the number of LPLs. In other studies, the density of LPLs per gram of the gut tissue had been estimated. Multiplying this density by the total weight of the human gut, the total number of LPLs was calculated, and the required percentage was found by dividing the total number of LPLs by the total number of lymphocytes in the body (found in [29]). Of note, many studies have estimated the density of T lymphocytes per unit area of the gut in several species of mammals (e.g. horses, monkeys, and humans). However, because of the difficult topological structure of the gut surface [25], it is difficult to translate the density of lymphocytes per unit of surface area to the total number of lymphocytes in the gut LP (results not shown).

\section{Estimated total numbers of lymphocytes per animal}

In rhesus macaques, there are $5.1 \times 10^{8}$ LPLs, which is $4 \%$ of the $1.2 \times 10^{10}$ lymphocytes estimated in an animal. In young pigs (10-12 weeks old) with, on average, $3.2 \times 10^{11}$ lymphocytes, the majority of lymphocytes, $44 \%$, were found in the thymus [44]. In the LP of their small intestines and in their PPs, $2.5 \%$ and $1.4 \%$, respectively, of the total body lymphocytes were found, and $17 \%$ of all lymphocytes were recovered from the MLNs. In adult pigs (14 months old), there are $3.5 \times 10^{9}$ LPLs per meter of the gut [45]. The total length of intestine in pigs is around 7-10 m [45], resulting in the total number of lymphocytes in the gut LP of adult pigs being $3.5 \times 10^{9} \times 8=2.8 \times 10^{10}$ cells. With the total number of lymphocytes in pigs estimated previously to be $3.2 \times 10^{11}$ cells [44], $9 \%$ of all lymphocytes in adult pigs are located in the gut LP. In young mice (4-8 weeks old), the majority of $\mathrm{CD}^{+} \mathrm{T}$ lymphocytes $(67 \%$ and $24 \%$, respectively) are found in the spleen and peripheral lymph nodes, whereas LP, PPs, and MLNs have lower cell percentages, i.e. $0.3 \%$, $0.2 \%$, and $4.5 \%$, respectively [46]. This results in $5 \%$ of all $T$ lymphocytes residing in the gut (LPLs + PPs + MLNs) of young mice.

Density of LPLs per gram of the gut tissue

Rothkotter [45] estimated a total of $3.5 \times 10^{7}$ LPLs per gram of gut tissue in pigs. The total weight of a human gut is $\sim 1.1 \mathrm{~kg}$ [47], and, if lymphocyte density in the LP is similar in humans and pigs, then the total number of LPLs in humans would amount to $3.5 \times 10^{7} \times 1.1 \times 10^{3}=3.85 \times 10^{10}$ or $3.85 \times 10^{10} / 4.6 \times 10^{11} \approx 8 \%$ of all lymphocytes in a human. In another study, a total of $7.7 \times 10^{6}$ lymphocytes per gram of gut tissue in humans was found [48]. Considering the above-described estimate of the weight of the human gut $(1.1 \mathrm{~kg}$ [47]), the total number of LPLs in humans is $7.7 \times 10^{6} \times 1.1 \times 10^{3}=8.5 \times 10^{9}$, which amounts only for $1.9 \%$ of the total body lymphocytes.

\section{Estimates for IELs}

There are only a few studies in which the total number of IELs has been estimated. Generally, the number of IELs is found to be similar to that of LPLs $[45,49,50]$. Rocha et al. [51] estimated a total of $3-4 \times 10^{7}$ IELs in a mouse, which is $\sim 40 \%-50 \%$ of the number of lymphocytes in the mouse spleen; however, others found at least 10 -fold fewer IELs [49,52]. In the future, the estimates of the total number of lymphocytes residing in the gut will need to be corrected by the number of IELs.

humans, macaques, pigs, and mice, suggests that only $1 \%-9 \%$ of all body lymphocytes are found in the gut LP, and that $5 \%-20 \%$ of all lymphocytes are located in the entire gut (Box 2 and Table 2). Taken together, these results suggest that spleen and lymph nodes, rather than the gut, are the major compartments of lymphocytes, including $\mathrm{CD}^{+}{ }^{+} \mathrm{T}$ lymphocytes (Table 3), in mammals. 
Table 2. Estimated percentage of lymphocytes residing in the gut in different species

\begin{tabular}{|c|c|c|c|c|}
\hline Species & LP (\%) & Gut lymphocytes (\%) & Comments & References \\
\hline Humans & 6.5 & 10.8 & Extrapolated from rats & {$[29]$} \\
\hline Humans & 8.0 & NA & Extrapolated from pigs & [45] \\
\hline Humans & 1.9 & NA & & [48] \\
\hline Monkeys & 4.0 & NA & & [42] \\
\hline Pigs & 2.5 & 20.6 & Young pigs (10-12 weeks old, 20-25 kg) & [44] \\
\hline Pigs & 9.0 & NA & Adult pigs (14 months old, $50 \mathrm{~kg}$ ) & [45] \\
\hline Mice & 0.3 & 5.0 & Estimate for $\mathrm{CD}^{+} \mathrm{T}$ lymphocytes & [46] \\
\hline
\end{tabular}

The numbers shown are percentages of lymphocytes per compartment (LP or the whole gut) relative to the total number of lymphocytes per organism; and "NA" stands for "not available." Gut lymphocytes include lymphocytes found in LP, PPs, ILFs, and MLNs. In some cases, to calculate the percentage, the estimate of the total number of lymphocytes $\left(4.6 \times 10^{11}\right)$ given in Table 1 was used. The average percentage of total body lymphocytes found in the LP is $4.6 \%$; the value in the whole gut is $12.1 \%$.

\section{Implications and issues for future research}

Currently, it is widely believed that a massive early depletion of $\mathrm{CD}^{+} \mathrm{T}$ cells from the gut LP during acute $\mathrm{HIV}$ infection is one of the main factors driving disease progression $[5,6,8,10,11,13,14,16]$. In part, this belief is due to a general consensus that most of $\mathrm{CD} 4^{+} \mathrm{T}$ lymphocytes in the body reside in the gut LP. The data reviewed here, however, suggest that LP lymphocytes constitute only a minor fraction of lymphocytes in the body (Tables 2,3). Massive depletion of a small fraction of total-body $\mathrm{CD} 4^{+}$ $\mathrm{T}$ cells is not by itself expected to be such an insult that it would predispose infected individuals to death from opportunistic infections. By contrast, the observed depletion of $\mathrm{CD}^{+} \mathrm{T}$ cells from other lymphoid tissues might have a larger impact on disease progression.

The importance of massive depletion of $\mathrm{LP} \mathrm{CD}^{+}$ $\mathrm{T}$ lymphocytes for disease progression has been questioned in several recent studies. SIV infection of sooty mangabeys (SMs) and african green monkeys (AGMs), which are natural hosts of SIVsm and SIVagm, respectively, also leads to a dramatic depletion of $\mathrm{LP} \mathrm{CD} 4^{+} \mathrm{T}$ lymphocytes $[32,33]$. However, infected animals remain typically healthy throughout life, despite a chronically high viral load $[32,33]$. Furthermore, infection of rhesus macaques (RMs) with SIVagm leads to a dramatic depletion of LP $\mathrm{CD}^{+}{ }^{+} \mathrm{T}$ lymphocytes as well, but these animals survive the infection and clear the virus [33]. Finally, it has been argued that because CCR5-expressing effector memory $\mathrm{CD}^{+} \mathrm{T}$ cells are produced by differentiation of central memory $\mathrm{CD}^{+} \mathrm{T}$ cells and have limited regenerative capacity [34,35], loss of these cells in HIV infection might be relatively unimportant in disease progression [35].

However, several studies have emphasized the potential importance of loss of $\mathrm{CD}^{+} \mathrm{T}$ cells from the gut LP for disease progression. Preservation of memory $\mathrm{CD} 4^{+} \mathrm{T}$ cells in the gut LP in vaccinated monkeys, after rechallenge with SIV, correlates with long-term survival [36,37]. Furthermore, SIV infection of macaques leads to an increased concentration of lipopolysaccharide (LPS) in the blood, which, in turn, is associated with high levels of immune activation [38]. LPS accumulation in blood might result from a translocation (leakage) of bacterial products from the gut [38]. Of note, no increase in LPS levels in the blood occurs in SMs and AGMs infected with their natural viruses, SIVsm and SIVagm, respectively $[32,33]$. The detrimental effects of SIV infection in macaques and HIV infection in humans might, therefore, not be directly due to depletion of $\mathrm{CD}^{+} \mathrm{T}$ cells from the gut LP, but indirectly owing to translocation of microbial products from the gut to the blood, leading to chronic immune activation [38] (see Box 3). Translocation of bacterial products is probably speeded up if the integrity of the gut is affected, for example, owing to a depletion of IELs or IgA-producing plasma B cells in the gut (Box 3).

In immunology, observations are typically presented in cell frequencies rather than in total cell numbers. Flow cytometry readily allows one to obtain reliable frequencies of subpopulations in the immune system. Calculating cell numbers is more difficult, however, and typically gives much more variable outcomes (e.g. [39]). This Opinion demonstrates how important it is to have estimates for the total cell numbers in different organs, and it emphasizes that one should aim for documenting total population sizes rather than population frequencies. One example is, again, from HIV infection. Whereas many studies (but not all, $[40,41]$ ) have documented the reduction in frequency of $\mathrm{CD}^{+} \mathrm{T}$ lymphocytes in the asymptomatic phase of HIV and SIV infection in some lymphoid tissues, such as tonsils and blood, the extent of the loss of the total number of $\mathrm{CD}^{+} \mathrm{T}$ cells in lymphoid organs such as lymph nodes and spleen has hardly been quantified (but, see [42]). Measuring population sizes should allow for a more quantitative understanding of

Table 3. Estimated numbers of $\mathrm{CD4}^{+}$and $\mathrm{CD}^{+} \mathrm{T}$ lymphocytes in different organs of humans

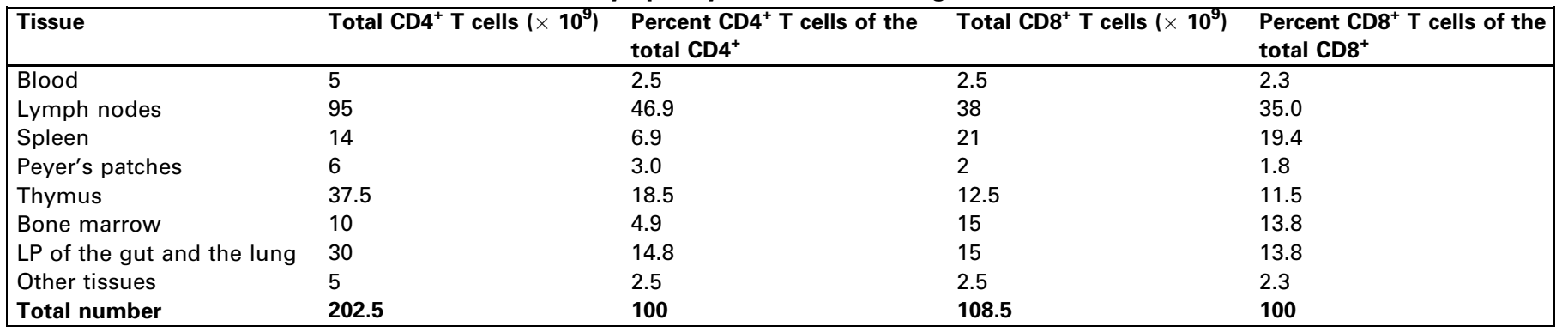

As in Table 1, the numbers are based on estimates of the total lymphocyte number in humans and the frequency of CD4 ${ }^{+}$and $\mathrm{CD} 8^{+} \mathrm{T}_{\text {cells }}$ in different tissues [31]. For the thymus, a ratio of 3:1 is assumed for CD4:CD8 T cells. For the row "Other tissues," we assumed that 70\% of cells are T cells, with a 2:1 ratio of CD4:CD8 T cells. As in Table 1, both peripheral and mucosal lymph nodes are included in the "Lymph nodes" row. 


\section{Box 3. Important questions for future research}

1. Total T-cell number and distribution. Studies estimating the number and phenotype of $T$ and $B$ lymphocytes in different organs of healthy humans or monkeys are desperately needed.

2. Number of T cells lost. Whereas the reduction in the frequency of $\mathrm{CD}^{+} \mathrm{T}$ cells in HIV and SIV infection in blood and the gut LP has been well documented, estimates of the total number of lymphocytes lost in acute HIV infection are still lacking.

3. Quantity versus quality. Whereas the fraction of the total body lymphocytes depleted in the acute phase of HIV infection is small, there could be a depletion of "high-quality" lymphocytes in the gut. These could be IELs, regulatory T cells (Tregs), and plasma B cells.

4. IELs. It has been shown that $\mathrm{CD} 8^{+}$IELs can be infected with HIV $[7,53]$. There is some contradictory data suggesting depletion [2] or an increase [54] of IELs by the AIDS phase in HIV-infected patients. Because IELs might determine the integrity of the gut cell wall, it remains to be investigated whether depletion of the total number of IELs occurs in HIV infection.

5. Tregs. Tregs play an important role in intestinal homeostasis [55], and several studies suggested that there is an increase in the fraction and the number of Tregs among intestinal $\mathrm{CD} 4^{+} \mathrm{T}$ cells after HIV or SIV infection $[56,57]$. An earlier study, however, claimed that there is a marked loss of Tregs $\left(\mathrm{CD} 25^{+} \mathrm{CD} 4^{+} \mathrm{T}\right.$ cells) in HIV-infected patients [58]. More studies are therefore needed to investigate the dynamics of the gut regulatory $\mathrm{CD}^{+} \mathrm{T}$ cells during HIV and SIV infection.

6. B cells. Several studies have found that many IgA-producing B cells reside in the gut LP in mice and humans $[27,28]$. In fact, it has been estimated that the total number of Ig-secreting B cells in the gut LP of mice accounts for more than $80 \%$ of all plasma $B$ cells in the body [28]. One study found no depletion of Ig-secreting B cells in HIV-infected men [1], but there was a depletion of plasma B cells in HIV-infected women [59]. It still needs to be determined whether plasma B cells are in fact selectively lost in HIV infection.

7. Macrophages and dendritic cells (DCs). Being part of the innate immune system, loss of CD4-expressing gut macrophages and DCs can impair the ability of B and T lymphocytes to mount immune responses. Several studies have found no reduction in the number of macrophages and loss of DCs in HIV-infected patients [59-61]. More studies are needed to determine which types of DCs are lost in HIV infection.

8. Repopulation. It takes several years of effective, highly active antiretroviral therapy (HAART) to restore normal numbers of $\mathrm{CD}^{+}{ }^{+}$LPLs in HIV-infected patients [18]. It is important to understand why homeostatic response to a loss of LPLs, which are a small fraction of total body lymphocytes, is not able to quickly restore the $\mathrm{CD}^{+} \mathrm{T}$-cell numbers in the gut $\mathrm{LP}$.

immunology, and it might ultimately allow for new insights into mechanisms of HIV pathogenesis.

\section{Acknowledgements}

We would like to thank David Masopust, Stephen Cose, and Frank Miedema for stimulating discussions; Stephen Cose for providing the data on T-cell numbers in mice; and Guido Silvestri with coauthors and Ivona Pandrea and Cristian Apetrei with coauthors for sharing their unpublished manuscripts. We would like to thank Christian Althaus, Jose Borghans, Daniel van der Post, Guido Silvestri, Joost Beltman, Frank Miedema, and two referees for the comments on previous versions of the manuscript. This work was supported by the Marie Curie Incoming International Fellowship (FP6) and Vici grant 016.048.603 from the Netherlands Organisation for Scientific Research (NWO).

\section{References}

1 Bishop, P.E. et al. (1987) Immunological study of the rectal mucosa of men with and without human immunodeficiency virus infection. Gut $28,1619-1624$
2 Lim, S.G. et al. (1993a) Loss of mucosal CD4 lymphocytes is an early feature of HIV infection. Clin. Exp. Immunol. 92, 448-454

3 Schneider, T. et al. (1995) Loss of CD4 T lymphocytes in patients infected with human immunodeficiency virus type 1 is more pronounced in the duodenal mucosa than in the peripheral blood. Berlin Diarrhea/Wasting Syndrome Study Group. Gut 37, 524-529

4 Clayton, F. et al. (1997) Selective depletion of rectal lamina propria rather than lymphoid aggregate CD4 lymphocytes in HIV infection. Clin. Exp. Immunol. 107, 288-292

5 Mehandru, S. et al. (2004) Primary HIV-1 infection is associated with preferential depletion of CD4+ T lymphocytes from effector sites in the gastrointestinal tract. J. Exp. Med. 200, 761-770

6 Brenchley, J.M. et al. (2004) CD4+ T cell depletion during all stages of HIV disease occurs predominantly in the gastrointestinal tract. J. Exp. Med. 200, 749-759

7 Smit-McBride, Z. et al. (1998) Gastrointestinal T lymphocytes retain high potential for cytokine responses but have severe CD4(+) T-cell depletion at all stages of simian immunodeficiency virus infection compared to peripheral lymphocytes. J. Virol. 72, 6646-6656

8 Veazey, R.S. et al. (1998) Gastrointestinal tract as a major site of CD4+ $\mathrm{T}$ cell depletion and viral replication in SIV infection. Science 280, $427-431$

9 Veazey, R.S. et al. (2000) Identifying the target cell in primary simian immunodeficiency virus (SIV) infection: highly activated memory CD4(+) $\mathrm{T}$ cells are rapidly eliminated in early SIV infection in vivo. J. Virol. 74, 57-64

10 Mattapallil, J.J. et al. (2005) Massive infection and loss of memory CD4+ T cells in multiple tissues during acute SIV infection. Nature 434, 1093-1097

$11 \mathrm{Li}$, Q. et al. (2005) Peak SIV replication in resting memory CD4+ T cells depletes gut lamina propria CD4+ T cells. Nature 434, 1148-1152

12 Picker, L.J. and Watkins, D.I. (2005) HIV pathogenesis: the first cut is the deepest. Nat. Immunol. 6, 430-432

13 Picker, L.J. (2006) Immunopathogenesis of acute AIDS virus infection. Curr. Opin. Immunol. 18, 399-405

$14 \mathrm{Hel}$, Z. et al. (2006) HIV infection: first battle decides the war. Trends Immunol. 27, 274-281

15 Brenchley, J.M. et al. (2006) HIV disease: fallout from a mucosal catastrophe? Nat. Immunol. 7, 235-239

16 Centlivre, M. et al. (2007) In HIV-1 pathogenesis the die is cast during primary infection. AIDS 21, 1-11

17 Veazey, R.S. and Lackner, A.A. (2006) Impact of antiretroviral therapy on intestinal lymphoid tissues in HIV infection. PLoS Med. 3, e515

18 Mehandru, S. et al. (2006) Lack of mucosal immune reconstitution during prolonged treatment of acute and early HIV-1 infection. PLoS Med. 3, e484

19 Cerf-Bensussan, N. and Guy-Grand, D. (1991) Intestinal intraepithelial lymphocytes. Gastroenterol. Clin. North Am. 20, 549-576

20 Kraehenbuhl, J-P. and Neutra, M.R. (1992) Molecular and Celluar Basis of Immune Protection of mucosal Surfaces. Phys. Rev. 72, 853-879

21 McDonald, T.T. and Spencer, J. (1994) General background. In Gastrointestinal and hepatic immunology (Heatley, R.H., ed.), pp. 123, Cambridge, Cambridge Univ. Press

22 Picker, L.J. et al. (2004) Insufficient production and tissue delivery of CD4+ memory T cells in rapidly progressive simian immunodeficiency virus infection. J. Exp. Med. 200, 1299-1314

23 Ho, D.D. et al. (1995) Rapid turnover of plasma virions and CD4 lymphocytes in HIV-1 infection. Nature 373, 123-126

24 Haase, A.T. (2005) Perils at mucosal front lines for HIV and SIV and their hosts. Nat. Rev. Immunol. 5, 783-792

25 Wilson, T.H. (1962) Intestinal absorption. W. B. Saunders Company, (Philadelphia, USA)

26 Wilson, J.P. (1967) Surface area of the small intestine in man. Gut 8 , 618-621

27 Brandtzaeg, P. (1989) Overview of the mucosal immune system. Curr. Top. Microbiol. Immunol. 146, 13-25

28 van der Heijden, P.J. et al. (1987) Contribution of immunoglobulinsecreting cells in the murine small intestine to the total 'background' immunoglobulin production. Immunology 62, 551-555

29 Trepel, F. (1974) Number and distribution of lymphocytes in man. A critical analysis. Klin. Wschr. 52, 511-515

30 Brandtzaeg, P. and Pabst, R. (2004) Let's go mucosal: communication on slippery ground. Trends Immunol. 25, 570-577 
31 Westermann, J. and Pabst, R. (1992) Distribution of lymphocyte subsets and natural killer cells in the human body. Clin. Investig. $70,539-544$

32 Gordon, S.N. et al. (2007) Severe depletion of mucosal CD4+ T cells in AIDS-free SIV-infected sooty mangabeys. J. Immunol. 179, 3026-3034

33 Pandrea, I. et al. (2007) Acute loss of intestinal CD4+ T-cells is not predictive of SIV virulence. J. Immunol. 179, 3035-3046

34 Sallusto, F. et al. (2004) Central memory and effector memory T cell subsets: function, generation, and maintenance. Annu. Rev. Immunol. $22,745-763$

35 Grossman, Z. et al. (2006) Pathogenesis of HIV infection: what the virus spares is as important as what it destroys. Nat. Med. 12, 289-295

36 Letvin, N.L. et al. (2006) Preserved CD4+ central memory T cells and survival in vaccinated SIV-challenged monkeys. Science 312, 1530-1533

37 Mattapallil, J.J. et al. (2006) Vaccination preserves CD4 memory T cells during acute simian immunodeficiency virus challenge. J. Exp. Med. 203, 1533-1541

38 Brenchley, J.M. et al. (2006b) Microbial translocation is a cause of systemic immune activation in chronic HIV infection. Nat. Med. 12, $1365-1371$

39 Althaus, C.L. et al. (2007) Dynamics of CD8+ T cell responses during acute and chronic lymphocytic choriomeningitis virus infection. J. Immunol. 179, 2944-2951

40 Rosenberg, Y.J. et al. (1993) Decline in the CD4+ lymphocyte population in the blood of SIV-infected macaques is not reflected in lymph nodes. AIDS Res. Hum. Retroviruses 9, 639-646

41 Rosok, B.I. et al. (1996) Reduced CD4 cell counts in blood do not reflect CD4 cell depletion in tonsillar tissue in asymptomatic HIV-1 infection. AIDS 10, F35-F38

42 Sopper, S. et al. (2003) Impact of simian immunodeficiency virus (SIV) infection on lymphocyte numbers and T-cell turnover in different organs of rhesus monkeys. Blood 101, 1213-1219

43 Kindred, J.E. (1942) A quantitative study of hemopoetic organs of young adult albino rats. Am. J. Anat. 71, 207-243

44 Pabst, R. and Trepel, F. (1975) Quantitative evaluation of the total number and distribution of lymphocytes in young pigs. Blut $31,77-86$

45 Rothkotter, H.J. et al. (1994) Lymphoid and non-lymphoid cells in the epithelium and lamina propria of intestinal mucosa of pigs. Gut 35 , 1582-1589

46 Cose, S. et al. (2006) Evidence that a significant number of naive T cells enter non-lymphoid organs as part of a normal migratory pathway. Eur. J. Immunol. 36, 1423-1433
47 Aiello, L.C. and Wheeler, P. (1995) The expensive-tissue hypothesis. The brain and the digestive system in human and primate evolution. Curr. Anthropol. 36, 199-221

48 Selby, W.S. et al. (1984) Intestinal lymphocyte subpopulations in inflammatory bowel disease: an analysis by immunohistological and cell isolation techniques. Gut 25, 32-40

49 Masopust, D. et al. (2001) Preferential localization of effector memory cells in nonlymphoid tissue. Science 291, 2413-2417

50 Ruthlein, J. et al. (1992) Anti-CD2 and anti-CD3 induced T cell cytotoxicity of human intraepithelial and lamina propria lymphocytes. Gut 33, 1626-1632

51 Rocha, B. et al. (1991) The V $\beta$ repertoire of mouse gut homodimeric $\alpha$ CD8+ intraepithelial T cell receptor $\alpha / \beta+$ lymphocytes reveals a major extrathymic pathway of $\mathrm{T}$ cell differentiation. J. Exp. Med. 173, 483-486

52 Guy-Grand, D. et al. (1978) The mouse gut T lymphocyte, a novel type of $\mathrm{T}$ cell. Nature, origin, and traffic in mice in normal and graft-versushost conditions. J. Exp. Med. 148, 1661-1677

53 Mattapallil, J.J. et al. (1998) Intestinal intraepithelial lymphocytes are primed for $\gamma$ interferon and MIP-1 $\beta$ expression and display antiviral cytotoxic activity despite severe CD4(+) T-cell depletion in primary simian immunodeficiency virus infection. J. Virol. 72, 6421-6429

54 Nilssen, D.E. et al. (1996) Intraepithelial $\gamma / \delta \mathrm{T}$ cells in duodenal mucosa are related to the immune state and survival time in AIDS. J. Virol. 70, $3545-3550$

55 Izcue, A. et al. (2006) Regulatory T cells suppress systemic and mucosal immune activation to control intestinal inflammation. Immunol. Rev. 212, 256-271

56 Epple, H.J. et al. (2006) Mucosal but not peripheral FOXP3+ regulatory $\mathrm{T}$ cells are highly increased in untreated HIV infection and normalize after suppressive HAART. Blood 108, 3072-3078

57 Boasso, A. et al. (2006) Do regulatory T-cells play a role in AIDS pathogenesis? AIDS Rev. 8, 141-147

58 Zeitz, M. et al. (1994) Cell differentiation and proliferation in the gastrointestinal tract with respect to the local immune system. Ann. N. Y. Acad. Sci. 733, 75-86

59 Olaitan, A. et al. (1996) The distribution of immunocompetent cells in the genital tract of HIV-positive women. AIDS 10, 759-764

60 Lim, S.G. et al. (1993b) Mucosal macrophage subsets of the gut in HIV: decrease in antigen-presenting cell phenotype. Clin. Exp. Immunol. 92, $442-447$

61 Knight, S.C. (1996) Bone-marrow-derived dendritic cells and the pathogenesis of AIDS. AIDS 10, 807-817

\section{How to re-use Elsevier journal figures in multimedia presentations}

It's easy to incorporate figures published in Trends, Current Opinion or Drug Discovery Today journals into your multimedia presentations or other image-display programs.

1. Locate the article with the required figure on ScienceDirect and click on the 'Full text + links' hyperlink

2. Click on the thumbnail of the required figure to enlarge the image

3. Copy the image and paste it into an image-display program

Permission of the publisher is required to re-use any materials from Trends, Current Opinion or Drug Discovery Today journals or from any other works published by Elsevier. Elsevier authors can obtain permission by completing the online form available through the Copyright Information section of Elsevier's Author Gateway at http://authors.elsevier.com. Alternatively, readers can access the request form through Elsevier's main website at:

www.elsevier.com/locate/permissions 